\title{
Efecto del silicio orgánico en el rendimiento de maracuyá (Passiflora edulis), cultivada en Somate-Sullana
}

\author{
Effect of organic silicon on the yield of passion fruit \\ (Passiflora edulis), cultivated in Somate- Sullana
}

iD Ricardo Antonio Peña Castillo $\square_{y}$ (D) Miguel Ángel Galecio Julca

Universidad Nacional de Piura. Piura, Perú

\begin{tabular}{llll}
\hline Recibido: 27/05/2018 & Revisado: 06/06/2019 & Aceptado: 16/06/2019 & Publicado: 30/06/2019 \\
\hline
\end{tabular}

\section{RESUMEN}

La investigación se realizó en una plantación de maracuyá (Passiflora edulis) instalada en la localidad de Somate Alto - Sullana; previamente se hizo el análisis del suelo. El objetivo de este estudio fue evaluar el efecto de silicio orgánico en el rendimiento, calidad y rentabilidad económica, para lo cual se empleó el diseño Bloques Completos al Azar.

La unidad experimental estuvo conformada por 12 plantas, evaluándose las 4 centrales para los parámetros de rendimiento y 2 para los parámetros de calidad. Los tratamientos fueron: T0 (Testigo sin silicio), T1 T2, T3 y T4 con 25, 50, 75 y 100 kg.ha-1 de silicio orgánico respectivamente. Las evaluaciones se realizaron hasta el quinto recojo de frutos con madurez comercial (tres primeros meses de cosecha); las conclusiones fueron:

- El mejor tratamiento para el rendimiento fue el T0 (testigo) con 10589,87 Kg.ha-1 de fruta, sin embargo, el T0 se comportó estadísticamente igual al resto de tratamientos en el parámetro pH de jugo y sólo superó al T2 (50 kg.ha-1 de silicio) en el volumen de jugo.

- Respecto a los sólidos solubles totales, el T0 fue superado estadísticamente por el resto de los tratamientos. - No existió diferencias significativas entre los tratamientos T3 (75 kg.ha-1 de silicio), T4 (100 kg.ha-1 de silicio) y T0 (testigo) en el peso de fruto y volumen de jugo. El Tratamiento testigo (T0), tuvo la más alta rentabilidad económica con una relación beneficio/ costo de 1,41.

Palabras clave: Silicio, rendimiento, cultivo de maracuyá.

\section{ABSTRACT}

The investigation was carried out in a passion fruit plantation (Passiflora edulis) installed in the village of Somate Alto - Sullana; previously the soil analysis was performed. The objective of this study was to evaluate the effect of organic silicon on yield, quality and economic profitability, for which the Random Complete Blocks design was used.

The experimental unit consisted of 12 plants, evaluating the 4 plants for the yield parameters and 2 for the quality parameters. The treatments were: T0 (control without silicon), T1 T2, T3 and $\mathrm{T} 4$ with 25, 50, 75 and $100 \mathrm{~kg}$.ha- 1 of organic silicon respectively. The evaluations were made until the fifth harvest of fruits with commercial maturity (first three months of harvest); 
The conclusions were:

- The best treatment for yield was T0 (control) with 10589,87 Kg.ha-1 of fruit, however, T0 behaved statistically equal to the rest of treatments in the juice $\mathrm{pH}$ parameter and only exceeded to T2 (50 kg.ha-1 of silicon) in the volume of juice. - Regarding total soluble solids, T0 was statistically exceeded by the rest of treatments.

- There were no significant differences between treatments T3 (75 kg.ha-1 of silicon), T4 (100 kg.ha- 1 of silicon) and T0 (control) in fruit weight and juice volume.

- The Control Treatment (TO), had the highest economic profitability with a benefit / cost ratio of 1,41 .

Keywords: Silicon, yield, passion fruit cultivation.

\section{INTRODUCCIÓN}

En la actualidad se conocen más de 400 variedades de maracuyá, siendo el Perú uno de los centros de origen de esta planta; algunas variedades se consumen en fresco, mientras que otras son más apreciadas por la industria dado su mayor porcentaje de acidez (Amaya, J. 2009).

En el país se cultiva las maracuyás amarillas y púrpuras, siendo Piura una de las regiones donde el área de cultivo se viene incrementando, de esta manera, el cultivo de maracuyá se ha convertido en uno de los cultivos alternativos al sembrío de arroz, dado la escasez de agua por ser ésta una zona árida y ante los embates del cambio climático se hace necesario la reconversión de cultivos.

En la región Piura, existen alrededor de 100 hectáreas instaladas de este cultivo, principalmente en la zona de Tambogrande, Valle de San Lorenzo, Somate y aledaños (Infoagro, 2009); la mayoría de productores son pequeños agricultores, quienes instalan áreas de cultivo relativamente pequeñas, donde la tecnología es muy precaria, no se realizan fertilizaciones, podas y labores culturales de manera oportuna, causas que repercute indudablemente en bajos rendimientos, mala calidad de la fruta, por lo que la rentabilidad es baja y el productor muchas veces opta por instalar otros cultivos.

Por otro lado, la extracción de nutrientes por parte de las cosechas, no se repone de manera adecuada y técnica, de tal manera que en los análisis de frutos se encuentran deficiencias nutricionales.

Al margen de los macro y micronutrientes comunes, la extracción de silicio activo de los suelos agrícolas por cada cosecha es, en promedio, de 40 a 300 kg.ha-1 (Quero, 2008), de tal modo que la fertilización es uno de los aspectos más importantes del cultivo del maracuyá porque de ella dependen la productividad, la calidad de los frutos, los costos de producción y rentabilidad.

El nivel de nutrientes en el suelo puede ser el origen de muchos desórdenes fisiológicos, pudiendo llegar a alterar la tasa respiratoria de los frutos (Pereira, 2015).

La fertilización con silicio puede optimizar el aprovechamiento del agua de riego en un 30 a $40 \%$ y ampliar los intervalos del riego sin efectos negativos sobre las plantas.

Además, el silicio incrementa la resistencia a la sequía en las plantas, adicionalmente al 
sistema irrigación - drenaje, la fertilización con minerales de silicio activo, permite completar la rehabilitación de suelos afectados por sales, compactación y bajos niveles de pH (Quero, 2008); una vez aplicado al suelo reacciona con el agua transformándose en ácido monosilícico (H4SiO4) moviéndose rápidamente a través del xilema (AGROMIL, 2006), por otra parte, se encuentra presente en los tejidos de la planta en cuatro formas: mineral, orgánica, polimérica y cristalina, ésta última se encuentra en la superficie de las hojas, proporcionándole brillo y formando parte de la estructura de los tricomas y fitolitos (Quero, 2008), bases fundamentales que lo constituyen como un elemento importante en la producción de los cultivos.

De acuerdo a ello, se planteó esta investigación, donde se evaluaron diferentes dosis de silicio orgánico, para poder establecer la de mejor respuesta en el rendimiento, calidad y rentabilidad, y poder brindar al agricultor una alternativa de mejora del cultivo.

\section{MATERIALES Y MÉTODOS}

\section{Análisis de suelos.}

Se realizó previo a la siembra, para ello se tomaron muestras de suelo a una profundidad de $30 \mathrm{~cm}$. de todo el campo experimental, luego se homogeneizaron y por la técnica del cuarteo se obtuvo una muestra representativa de un kilo.

El análisis físico - químico se realizó en el Laboratorio de Suelos de la Universidad Nacional de Piura.

\section{Condiciones de cultivo y cosecha Dosis/tratamientos}

Los tratamientos al suelo se realizaron con el producto comercial INTENSO SILICIO ORGÁNICO, el primer momento de aplicación fue a los 30 días después de la siembra en campo definitivo (30\% de la dosis), el segundo dos meses después (30\% de la dosis) y el tercero, dos meses después del segundo (40\% restante de la dosis). Ver Tabla 1.

Tabla 1. Tratamientos en estudio

\begin{tabular}{ccc}
\hline $\mathbf{N}^{\circ}$ & Tratamientos & Clave \\
\hline 1 & Sin aplicación de silicio & T0 \\
2 & $25 \mathrm{~kg} \cdot \mathrm{ha}^{-1}$ de silicio & T1 \\
3 & $50 \mathrm{~kg} \cdot \mathrm{ha}^{-1}$ de silicio & T2 \\
4 & $75 \mathrm{~kg} \cdot \mathrm{ha}^{-1}$ de silicio & T3 \\
5 & $100 \mathrm{~kg} \cdot \mathrm{ha}^{-1}$ de silicio & T4 \\
\hline
\end{tabular}

\section{Análisis estadístico}

Comprendió el análisis de Varianza (ANVA) para determinar la significación entre los tratamientos, además la prueba de Duncan al 0.05 de probabilidad.
Se empleó el diseño experimental Bloques Completos Al Azar (B.C.A). Se estudiaron 4 tratamientos con fertilizante Silicio orgánico más un testigo sin aplicación. 
El número de repeticiones fue de 3 , haciendo un total de 15 unidades experimentales. Cada unidad experimental estuvo conformada por 12 plantas, evaluándose las dos centrales para los parámetros de calidad y diámetro ecuatorial (6 plantas por tratamiento), mientras que para los parámetros que tienen relación directa con el rendimiento se evaluaron 4 plantas centrales por unidad experimental (12 plantas por tratamiento), además, un tratamiento estuvo separado de otro por dos hileras de plantas para evitar posibles interacciones entre ellos.

\section{Parámetros de rendimiento}

Se realizaron en el mismo campo experimental y fueron: Número de frutos por planta, peso de fruto (g), diámetro ecuatorial de fruto (cm) y rendimiento $(\mathrm{kg} / \mathrm{planta})$.

\section{Indicadores de calidad}

Se determinaron con los frutos de la quinta cosecha, para ello fueron trasladados al laboratorio de frutos y semillas del departamento académico de agronomía y fitotecnia de la universidad Nacional de Piura, se determinó: volumen de jugo (se extrajo las semillas más arilo, se separó el jugo mediante el uso de coladores de laboratorio y se midió en un vaso graduado en $\mathrm{cc}$ ); $\mathrm{pH}$ (se usó el potenciómetro o peachímetro, mediante el método de ensayo: NMX-F-317NORMEX-2013) y sólidos solubles totales (expresado en ${ }^{\circ}$ Brix con refractómetro manual, método de ensayo NMX-F-103NORMEX-2010).

\section{RESULTADOS}

\section{Análisis del suelo del campo experimental}

En la Tabla 2, se muestran los resultados del análisis físico - químico del suelo. Clase textural Franco Arcilloso, con $38 \%$ de arena, $31 \%$ de limo y $31 \%$ de arcilla, es un suelo de textura media, adecuado para el cultivo de maracuyá, conforme lo establece (Malavolta, 1994).

El maracuyá se desarrolla de la mejor manera en suelos con pH mayor a 5 (Navarro, 2003), esta afirmación coincide con nuestro resultado donde el $\mathrm{pH}$ es moderadamente alcalino, con un valor de 8,08 característico de los suelos de la zona de Somate Alto y que en términos generales no representa problema para el cultivo de maracuyá excepto, posiblemente, en la disponibilidad de algunos micronutrientes, sin embargo, no hubo problemas en cuanto a estos nutrientes. Respecto a la fertilidad del suelo, la materia orgánica con un valor de 0,42 \%, se considera bajo de acuerdo con los valores de este parámetro; el nitrógeno, se registró con un valor muy bajo de $0,02 \%$; el fósforo disponible en un nivel medio con un valor de 9 ppm; por otro lado, el contenido de potasio asimilable fue de 180 ppm (nivel medio).

Considerando estos resultados, los niveles de nitrógeno y materia orgánica bajos son propios de los suelos calcáreos (FAO, 2019) es por ello que el productor realizó aplicaciones de urea de manera fraccionada durante la época de crecimiento de la vegetación para satisfacer la demanda de nitrógeno durante la época de crecimiento de la vegetación y fosfato diamónico al inicio del periodo vegetativo dada la lenta descomposición y poca movilidad del elemento debido a su inactividad en suelos alcalinos (Garrido. S, 1993).

La conductividad eléctrica, con un valor de 0,91 dS.m-1, es baja y no representó problemas de salinidad para el cultivo.

La capacidad de intercambio catiónico (C.I.C) fue de $23,17 \mathrm{cmol}(+) . \mathrm{Kg}-1$, se considera 
como un valor medio a alto, lo que indica que el suelo tiene un gran potencial para retener e intercambiar nutrientes, esto motivado por la presencia de arcillas del suelo (INTAGRI, 2019).

El Calcáreo $\left(\mathrm{CaCO}_{3}\right)$ fue de $5,11 \%$, lo que se considera un nivel alto que puede traer deficiencias de microelementos como Zn, B y $\mathrm{Fe}(\mathrm{FAO}, 2019) ;$ los mismos que fueron suplidos con la aplicación de abonos foliares por parte del productor. Los cationes $\mathrm{Ca}++, \mathrm{Mg}++, \mathrm{Na}+$ y $\mathrm{K}+$, tuvieron valores de 19,40; 2,90; 0,43 y 0,44 cmol (+). $\mathrm{Kg}-1$ respectivamente.

Estos valores nos permiten determinar un elevado porcentaje de saturación de bases, lo que indica que el suelo se encuentra saturado en bases y sus sedes de intercambio están siendo utilizadas (Garrido, S. 1993), la cantidad y disponibilidad de estos cationes intercambiables se encuentra asegurada en el suelo dado al nivel alto de capacidad de intercambio catiónico (INTAGRI, 2019).

Tabla 2. Resultados del análisis físico - químico del campo experimental

\begin{tabular}{|c|c|c|c|}
\hline Determinación & Unidad & Método & Resultado \\
\hline Clase textural & & Bouyoucus & Franco Arcilloso \\
\hline Arena & $\%$ & & 38,0 \\
\hline limo & $\%$ & & 31,0 \\
\hline Arcilla & $\%$ & & 31,0 \\
\hline $\mathrm{pH}(1: 2.5)$ & & Potenciómetro & 8,08 \\
\hline Materia orgánica & $\%$ & Walkley y Black & 0,42 \\
\hline Nitrógeno total & $\%$ & Estimado a partir de mat. orgánica & 0,02 \\
\hline Calcáreo $\left(\mathrm{CaCO}_{3}\right)$ & $\%$ & Volumétrico & 5,11 \\
\hline Fósforo disponible & $\mathrm{ppm}$ & Olsen & 9,0 \\
\hline Potasio asimilable & $\mathrm{ppm}$ & Espectrofotometría & 180,0 \\
\hline C.E ${ }^{*}$ & dS. $m^{-1}$ & Sumatoria de bases cambiables & 0,91 \\
\hline C.I.C** & $\mathrm{cmol}(+) . \mathrm{Kg}^{-1}$ & Acetato de amonio & 23,17 \\
\hline $\mathrm{Ca}^{++}$ & $\mathrm{cmol}(+) \cdot \mathrm{Kg}^{-1}$ & Complejométrico & 19,40 \\
\hline $\mathrm{Mg}^{++}$ & $\mathrm{cmol}(+) \cdot \mathrm{Kg}^{-1}$ & Complejométrico & 2,90 \\
\hline $\mathrm{Na}^{+}$ & $\mathrm{cmol}(+) \cdot \mathrm{Kg}^{-1}$ & Complejométrico & 0,43 \\
\hline $\mathrm{K}^{+}$ & $\mathrm{cmol}(+) . \mathrm{Kg}^{-1}$ & complejométrico & 0,44 \\
\hline
\end{tabular}

${ }^{*}$ C.E: Conductividad eléctrica; ${ }^{* *}$ C.I.C: Capacidad de intercambio catiónico

\section{Número de frutos por planta de maracuyá}

Se determinó en frutos que alcanzaron el grado de madurez comercial durante las cinco primeras cosechas, el análisis de varianza, indica que para efectos de bloques existe alta significación estadística, no existiendo diferencias significativas entre los tratamientos, el coeficiente de variación fue de $11,70 \%$.

\section{Efecto de tratamientos}

La prueba al 0,05 (Tabla 3), no detecta diferencias 
estadísticas entre los tratamientos evaluados. Los valores promedio para este parámetro, fluctúan entre 45,67 y 64,00 frutos por planta correspondientes a los tratamientos $T 1(25 \mathrm{Kg}$ Si.ha-1) y T0 (sin aplicación) respectivamente.

\section{Peso promedio de un fruto de maracuyá}

El análisis de varianza, indica que existen diferencias significativas entre los tratamientos siendo el coeficiente de variabilidad de $5,98 \%$.

Tabla 3. Efecto de los tratamientos sobre el número de frutos/planta de maracuyá

\begin{tabular}{|c|c|c|}
\hline Tratamiento & Promedio $\mathrm{n}=$ frutos/planta & Duncan 0,05 \\
\hline T0 (Sin aplicación) & 64,00 & a \\
\hline T2 (50 Kg Si.ha $\left.{ }^{-1}\right)$ & 55,83 & a \\
\hline T3 (75 Kg Si.ha $\left.{ }^{-1}\right)$ & 52,83 & a \\
\hline T4 (100 Kg Si.ha-1) & 52,00 & a \\
\hline T1 (25 Kg Si.ha-1) & 45,67 & a \\
\hline
\end{tabular}

\section{Efecto de tratamientos}

La prueba al 0.05 (Tabla 4), establece que los tratamientos T3 (75 Kg Si.ha- 1$)$, T4 (100 Kg Si.ha-1), T0 (Sin aplicación) y $\mathrm{T} 2(75 \mathrm{Kg} \mathrm{Si}$.ha-1) con peso de fruto de $175,77,169,33,165,47$ y 159,33 g., respectivamente, son estadísticamente iguales entre sí, pero sólo los tratamientos T3, T4 y T0 superan al tratamiento T1 (25 Kg Si.ha-1).

Tabla 4. Efecto de los tratamientos sobre el peso de un fruto de maracuyá.

\begin{tabular}{|c|c|c|}
\hline Tratamiento & Peso promedio frutos/planta (g) & Duncan 0,05 \\
\hline T3 (75 Kg Si.ha $\left.{ }^{-1}\right)$ & 175,77 & $\mathrm{a}$ \\
\hline T4 (100 Kg Si.ha $\left.{ }^{-1}\right)$ & 169,33 & a \\
\hline T0 (Sin aplicación) & 165,47 & a \\
\hline T2 (50 Kg Si.ha $\left.{ }^{-1}\right)$ & 159,33 & $a b$ \\
\hline T1 (25 Kg Si.ha $\left.{ }^{-1}\right)$ & 145,60 & $\mathrm{~b}$ \\
\hline
\end{tabular}




\section{Rendimiento de maracuyá en Kg.ha-1}

Tabla 5. Rendimiento de maracuyá en Kg. ha-1.

\begin{tabular}{|c|c|c|c|c|c|}
\hline Tratamiento & $\begin{array}{c}\text { № de } \\
\text { frutos/pta }\end{array}$ & $\begin{array}{l}\text { Peso prom. } \\
\text { De fruto (kg) }\end{array}$ & $\begin{array}{c}\text { Peso frutos/pta. } \\
\text { (kg) }\end{array}$ & $\begin{array}{c}\text { № } \\
\text { plantas/ha }\end{array}$ & $\begin{array}{c}\text { Rdto. } \\
\left(\mathbf{k g} \cdot \mathrm{ha}^{-1}\right)\end{array}$ \\
\hline T0 (Sin aplicación) & 64,00 & 0,16547 & 10,58987 & 1000 & 10589,87 \\
\hline T1 (25 Kg Si.ha ${ }^{-1}$ ) & 45,67 & 0,14560 & 6,64955 & 1000 & 6649,55 \\
\hline T2 (50 Kg Si.ha ${ }^{-1}$ ) & 55,83 & 0,15933 & 8,89558 & 1000 & 8895,58 \\
\hline T3 (75 Kg Si.ha-1) & 52,83 & 0,17577 & 9,28575 & 1000 & 9285,75 \\
\hline T4 (100 Kg Si.ha $\left.{ }^{-1}\right)$ & 52,00 & 0,16933 & 8,80533 & 1000 & 8805,33 \\
\hline
\end{tabular}

*Basado en frutos que alcanzaron madurez comercial, durante las cinco primeras cosechas.

${ }^{* *}$ Calculado en base al distanciamiento: $4 \mathrm{~m}$ entre plantas, $5 \mathrm{~m}$ entre surcos, dispuestos en surcos mellizos.

\section{Diámetro ecuatorial en frutos de maracuyá}

El análisis de varianza, reporta que no existen diferencias significativas para efectos de bloques, ni entre los tratamientos, referente a la observación diámetro ecuatorial de frutos de maracuyá; el coeficiente de variación fue de $5,15 \%$.

\section{Efecto de tratamientos}

La prueba al 0,05 (Tabla 6), establece que no existen diferencias significativas entre los tratamientos, los cuales se comportan estadísticamente igual.

Los valores promedio varían entre 6,97 y 7,33 $\mathrm{cm}$., correspondientes a los tratamientos T4 (100 Kg Si.ha-1) y T0 (sin aplicación) respectivamente.

Tabla 6. Efecto de los tratamientos sobre el diámetro ecuatorial en maracuyá.

\begin{tabular}{|c|c|c|}
\hline Tratamiento & Promedio diámetro de fruto $(\mathrm{cm})$ & Duncan 0,05 \\
\hline TO (Sin aplicación) & 7,33 & $a$ \\
\hline T2 (50 Kg Si.ha $\left.{ }^{-1}\right)$ & 7,21 & a \\
\hline T1 (25 Kg Si.ha $\left.{ }^{-1}\right)$ & 7,10 & a \\
\hline T3 (75 Kg Si.ha $\left.{ }^{-1}\right)$ & 7,06 & a \\
\hline T4 (100 Kg Si.ha $\left.{ }^{-1}\right)$ & 6,97 & a \\
\hline
\end{tabular}

\section{Volumen de jugo/fruto de maracuyá (ml)}

El análisis de varianza indica que no existen diferencias significativas para efectos de bloques, mientras que, para los tratamientos en estudio si existe significación estadística; el coeficiente de variación fue de 12,61\%.

\section{Efecto de tratamientos}

La prueba al 0,05 (Tabla 7), establece que los tratamientos T3 (75 Kg Si.ha-1) con un promedio de volumen de jugo/fruto de 50,67 $\mathrm{ml}$, se comporta estadísticamente igual a los tratamientos T4 (100 Kg Si.ha-1) y T0 (Sin 
aplicación) con valores numéricos de 48,67 y $44,33 \mathrm{ml}$ de jugo/fruto respectivamente, pero solo el tratamiento T3 supera estadísticamente al tratamiento T2 (50 Kg Si.ha-1).

Tabla 7. Efecto de los tratamientos sobre promedio de volumen de jugo/fruto (ml)

\begin{tabular}{|c|c|c|}
\hline Tratamiento & Prom. volumen jugo/fruto (ml) & Duncan 0,05 \\
\hline T3 (75 Kg Si.ha-1) & 50,67 & $a$ \\
\hline T4 (100 Kg Si.ha-1) & 48,67 & $a b$ \\
\hline T0 (Sin aplicación) & 44,33 & $a b$ \\
\hline T1 (25 Kg Si.ha-1) & 30,33 & bc \\
\hline T2 (50 Kg Si.ha-1) & 26,00 & c \\
\hline
\end{tabular}

\section{Sólidos solubles totales ( ${ }^{\circ} \mathrm{Brix}$ )}

El análisis de varianza, indica que no existen diferencias significativas tanto para efecto de bloques como para los tratamientos en estudio; el coeficiente de variación fue de 1,50\%.

Efecto de tratamientos

La prueba al 0,05 (Tabla 8), establece que los tratamientos T4 (100 Kg Si.ha- 1 ), T1 (100 Kg Si.ha-1), T3 (100 Kg Si.ha-1) y $\mathrm{T} 2$ (100 Kg Si.ha-1), se comportan estadísticamente igual, pero solo los tratamientos T4 y T1 superan al T0 ( $\sin$ aplicación) con el que se obtuvo un promedio de $15,67^{\circ}$ Brix.

Tabla 8. Efecto de tratamientos sobre sólidos solubles totales, maracuyá ('Brix)

\begin{tabular}{lcc}
\hline Tratamiento & Promedio sólidos solubles totales ( ${ }^{\circ}$ Brix) & Duncan 0,05 \\
\hline T4 (100 Kg Si.ha $\left.{ }^{-1}\right)$ & 16,27 & $\mathrm{a}$ \\
T1 $\left(25 \mathrm{Kg} \mathrm{Si}_{\text {.ha }}{ }^{-1}\right)$ & 16,17 & $\mathrm{a}$ \\
T3 $\left(75 \mathrm{Kg} \mathrm{Si}^{-1} \mathrm{~h}^{-1}\right)$ & 16,10 & $\mathrm{ab}$ \\
T2 $\left(50 \mathrm{Kg} \mathrm{Si}^{-1}\right.$ ) & 16,00 & $\mathrm{ab}$ \\
T0 (Sin aplicación) & 15,67 & $\mathrm{~b}$ \\
\hline
\end{tabular}

\section{Ph en fruta de maracuyá}

El análisis de varianza, indica que existen diferencias altamente significativas para el efecto bloque, sin embargo, para los tratamientos en estudio no existen diferencias significativas; el coeficiente de variabilidad fue de $1,63 \%$.

\section{Efecto de tratamientos}

La prueba al 0,05 (Tabla 9), establece que no existen diferencias significativas entre los tratamientos. Los valores promedio varían entre 3,17 y 3,28, correspondientes a los tratamientos T4 (100 Kg Si.ha- 1$)$ y T3 $(100 \mathrm{Kg}$ Si.ha-1) respectivamente. 
Tabla 9. Efecto de los tratamientos sobre el pH en fruta de maracuyá

\begin{tabular}{|c|c|c|}
\hline Tratamiento & Promedio ph & Duncan 0,05 \\
\hline T3 (75 Kg Si.ha-1) & 3,28 & a \\
\hline T2 (50 Kg Si.ha-1) & 3,24 & a \\
\hline T1 (25 Kg S.ha-1) & 3,22 & a \\
\hline T0 (Sin aplicación) & 3,21 & a \\
\hline T4 (100 Kg Si.ha $\left.{ }^{-1}\right)$ & 3,17 & a \\
\hline
\end{tabular}

\section{Análisis económico}

De acuerdo a la Tabla 10, se deduce que tratamiento de más alta rentabilidad fue el T0 (testigo sin aplicación), con una relación beneficio - costo de 1,41, este tratamiento corresponde también al de mayor rendimiento en Kg.ha-1, esto quiere decir que por cada sol invertido se obtienen 1,41 soles de utilidad.

En segundo lugar, se ubica el tratamiento T3 que corresponde a la aplicación de $75 \mathrm{~kg}$.ha-1 del producto comercial Intenso - silicio orgánico, con una relación beneficio-costo de 0,97 . Este tratamiento tiene el segundo mejor rendimiento en Kg.ha-1, lo que significa que por cada sol invertido se obtienen 0,97 soles de utilidad.

La menor relación beneficio - costo corresponde al tratamiento $\mathrm{T} 1$, que corresponde a la dosis de $25 \mathrm{~kg}$ del producto comercial Intenso - Silicio orgánico.

Tabla 10. Análisis económico para el cultivo de maracuyá.

\begin{tabular}{|c|c|c|c|c|c|c|}
\hline Trat. & $\begin{array}{l}\text { Costo } \\
\text { silicio } \\
\text { S/. }\end{array}$ & $\begin{array}{l}\text { Costo } \\
\text { producción S/. }\end{array}$ & $\begin{array}{c}\text { Rendimiento } \\
\mathrm{kg} / \mathrm{ha}\end{array}$ & $\begin{array}{c}\text { Valor bruto } \\
\text { S/. }\end{array}$ & $\begin{array}{c}\text { Utilidad } \\
\text { S/. }\end{array}$ & $\begin{array}{c}\text { Relación } \\
\text { b/c* }\end{array}$ \\
\hline TO & 0,00 & 3850,00 & 10589,87 & 9266.14 & 5416,14 & 1,41 \\
\hline T1 & 90,00 & 3940,00 & 6649,55 & 5818.36 & 1878,36 & 0,48 \\
\hline $\mathrm{T} 2$ & 180,00 & 4030,00 & 8895,58 & 7783.63 & 3753,63 & 0,93 \\
\hline T3 & 270,00 & 4120,00 & 9285,75 & 8125.03 & 4005,03 & 0,97 \\
\hline T4 & 360,00 & 4210,00 & 8805,33 & 7704.66 & 3494,66 & 0,83 \\
\hline $\begin{array}{l}\text {. } \\
. \\
. \\
*\end{array}$ & \multicolumn{3}{|c|}{$\begin{array}{l}\text { Costo de producción: } \\
\text { Costo de saco de INTENSO -SILICIO } \\
\text { Precio de venta de maracuyá } \\
\text { Precio de venta de maracuyá }\end{array}$} & \multicolumn{2}{|c|}{$\begin{array}{l}\text { S/. } 3850,00 \\
\text { S/. } 90,00 \text { sacox } 25 \mathrm{Kg} . \\
\text { S/. } 35,00 \\
\text { S/. } 0,875\end{array}$} & \\
\hline
\end{tabular}




\section{DISCUSIÓN}

En los parámetros de rendimiento, el tratamiento T0 (testigo sin aplicación) presentó el mayor número de frutos/planta y diámetro ecuatorial por fruto; numéricamente supera al resto de tratamientos, sin embargo, estadísticamente se comportan de manera similar.

Estos datos van a repercutir finalmente en el rendimiento, donde el tratamiento testigo reportó $10589,87 \mathrm{~kg}$. de fruta de maracuyá por hectárea, seguido de cerca por el tratamiento T3 $(75 \mathrm{Kg}$. Silicio/Ha) con 9285,75 kg.ha-1 , esto último, probablemente se debe a que el silicio influye en ciertas características morfológicas tales como materia seca, lográndose incrementos altamente significativo hasta de 34,9\% para dosis de $100 \mathrm{mg} . \mathrm{kg}-1$ de silicio y se asocia a un mejor índice de materia seca radicular $(0,17)$ para la misma dosis respecto a la no aplicación de Si, lo cual implica una mayor capacidad de absorción de nutrientes (Borda, et al., 2007), así como mayor número de hojas, y la relación de crecimiento entre raíz, parte aérea y diámetro de tallo se ve muy favorecida (Caicedo y Chavarriaga, 2007).

Los efectos del sílice han sido relacionados con: eficiencia en el uso del agua, incremento en los rendimientos del cultivo y translocación del fósforo (Primavesi, 1984), de allí que Carlos, (2018) obtuvo rendimientos promedios de 10950,00 kg.ha-1 y rentabilidad de US\$3041,00 al aplicar silicio en mezcla con $\mathrm{N}+\mathrm{K}+\mathrm{B}+$ ácido giberélico, datos muy cercanos a los obtenidos con el tratamiento T3 $(75 \mathrm{Kg}$ Silicio.ha-1) en este estudio.

A pesar de que el testigo sin aplicación de silicio superó en rendimiento al resto de tratamientos, es el tratamiento T3, el que reporta numéricamente el mayor peso promedio de un fruto en madurez comercial (175,77 gr/fruto), aunque estadísticamente se comporta igual con el control $(165,47 \mathrm{gr} /$ fruto).

En los parámetros de calidad; los frutos tratados con el T3 (75 Kg Silicio.ha- 1$)$, reportan el mayor volumen promedio de jugo con un valor de 50,67 ml de jugo/fruto, superando al testigo y resto de tratamientos incluso en los parámetros: solidos solubles totales (16,27 ${ }^{\circ}$ Brix) y 3,28 de pH de jugo de fruta, datos que son muy coincidentes con los obtenidos por (Rodríguez, et al., 2015) quienes obtuvieron 16,3 ${ }^{\circ}$ Brix en frutos de maracuyá en madurez comercial, 4,30 de acidez titulable y 3,80 de $\mathrm{pH}$; de igual manera (Barrientos, 2014) encontró 14,8 \% de solidos solubles, pH 3,25 y acidez 4,8\% en frutos de maracuyá al estado maduro.

Cabe indicar que la parcela donde se instaló el cultivo está ubicada dentro de una zona donde el agua de regadío vía canal retorna con intervalos de 20 días, periodo que se torna muy estresante para la plantación, dadas las elevadas temperaturas y niveles de evapotranspiración altos, es posible que la aplicación de silicio tenga repercusión en el volumen de jugo que reporta el tratamiento T3 (75 Kg Silicio.ha-1) respecto al testigo sin aplicación, dado que la fertilización con silicio puede optimizar el aprovechamiento del agua de riego en un 30 a $40 \%$ y ampliar los intervalos del riego sin efectos negativos sobre las plantas, además, el silicio incrementa la resistencia a la sequía en las plantas (Quero, 2008).

\section{CONCLUSIONES}

El mejor rendimiento se obtuvo con el T0 testigo sin aplicación (10589,87 Kg.ha-1 de fruta de maracuyá en madurez comercial 
correspondiente a las cinco primeras cosechas), sin embargo, en $\mathrm{pH}$ de jugo se comportó estadísticamente igual al resto de tratamientos y sólo superó al tratamiento T2 (50 kg.ha-1 de silicio) en volumen de jugo. Respecto a los sólidos solubles totales ('Brix), el To fue superado estadísticamente por el resto de tratamientos, mientras que para los parámetros peso de fruto y volumen de jugo no existió diferencias significativas entre los tratamientos T3 (75 Kg Si.ha- 1$)$, T4 (100 Kg Si.ha-1) y T0, siendo este último el tratamiento de más alta rentabilidad económica, con una relación beneficio/costo de 1,41, lo cual está relacionado directamente con el rendimiento de fruta e indica la poca influencia de la aplicación de sílice en los diferentes tratamientos.

\section{REVISIÓN BIBLIOGRÁFICA}

Agribusines Asistencia Agroempresarial (1992). Manual técnico de maracuyá. Quito, Ecuador. 33 p.

AGROMIL (2006). Beneficio del Silicio para la Agricultura. Disponible en: http:// silicioagromil.com/site/2-uncategorised/49linea-edafica.html. México.

Amaya, J. (2009). El cultivo del maracuyá (Passiflora edulis form. Flavicarpa). Gerencia Regional Agraria La Libertad, Trujillo-Perú. 2009, 30p.

Barrientos, N. (2014). Formulación, evaluación organoléptica y físico- química de una mermelada mixta a base de loche (Cucurbita maxima Dutch) y maracuyá (Passiflora edulis). Rev. Ingeniería: Ciencia, Tecnología e Innovación VOL 1/N² 2, ISSN: 2313 -1926/dic. 2014.

Borda, O., Barón, F., y Gómez, M. (2007). El silicio como elemento benéfico en avena forrajera (Avena sativa L.): Respuestas fisiológicas de crecimiento y manejo. Agronomía Colombiana, v.25, n. 2, p. 273279, jul. 2007. ISSN 2357-3732. Disponible en: htpps://revistas.unal.edu.co/index.php/ agrocol/article/view/14130. Fecha de acceso: 22 abr. 2019.

Brady, N. (1992). The naturale and properties of soil. 10 ed. New York: Macmillan Publishing. $750 \mathrm{p}$.

Caicedo, L., y Chavarriaga, W. (2007). Efecto de la aplicación de dosis de silicio sobre el desarrollo en almácigo de plántulas de café variedad Colombia. Agron. 15(1): 27 - 37, 2007. ISSN 0568-3076. http://www. nuprec.com/Nuprec_Sp_archivos/CAFE/ CAFETO_archivos/Literatura\%20Cafe/ Silicio/Silicio\%20en\%20caf\%C3\%A9\%20.pdf. Consultado el 22 de abril del 2019.

CAJAMAR, (2014). Parámetros de calidad interna de hortalizas y frutas en la industria agroalimentaria. Negocio alimentario y cooperativo, publicación $N^{a}$ 005. Disponible en: http://chilorg.chil.me/downloaddoc/86426

Carlos, J. (2018). Efecto de la aplicación de N, K, B, Si y GA3 sobre el rendimiento del fruto de maracuyá Passiflora edulis F.v variedad INIAP-2009 en el cantón Daule. Facultad de Ciencias Agrarias Universidad de Guayaquil.

CENTRO INTERNACIONAL PARA LA AGRICULTURA TROPICAL - CIAT. (1985). Química de los suelos inundados. Investigación y producción de arroz. Cali: CIAT.

Deuman, y Ambiente, M. (2011). www. deuman.com. Recuperado el 24 de Julio de 2013. 
Dulanto, J., y Aguilar, M. (2011). Guía técnica: Manejo integrado de producción y sanidad de maracuyá en Piura. Universidad Nacional Agraria La Molina. P. 37

Epstein, E. (1999). "Silicon". En: Annual Review Plant Physiology and Plant Molecular Biology. Vol. 50. pp. 641-64.

FAO. (2019). El manejo de suelos calcáreos. Portal de suelos de laFAO, disponible en: http:// www.fao.org/soils-portal/soil-management/ manejo-de-suelos-problematicos/sueloscalcareos/es/

Fernández, E. (2015). Uso de silicio e Inductores de Resistencia en Relación a Huanglongbing (HLB) en Limón Persa (Citrus latifolia) y limón mexicano (Citrus aurantifolia). Tesis que para Obtener el Grado de Maestro en Ciencias en Biosistemática y Manejo de Recursos Naturales y Agrícolas Zapopan - Jalisco Universidad de Guadalajara -México.

Furcal, P., y Herrera, A. (1998). Efecto del silicio y plaguicidas en la fertilidad del suelo y rendimiento del arroz. Agron. Mesoam vol.24. $N^{0}$ 2. San Pedro Jul./ Dec. 2013. On-line versión ISSN 2215-3608.

Garrido, S. (1993). Interpretación de análisis de suelos. Hojas divulgativas Numero 5/93 HD. I.S.B.N.: 84-341-0810O. N.LP.O.: 253-94-007-5 - Depósito legal: M. 29.238-1994 (40.000 ejemplares). Imprime: Rivadeneyra, S. A. -Getafe (Madrid) - España.

Horna, R. (2007). Silicio en la Producción Agrícola. Recuperado el 23 de Julio de 2013, de Silicio en la Producción Agrícola: www.uteq.edu.ec/eventos/2007/congreso_ biotecnologia/832pdf
INFOAGRO. (2009). Aumenta producción de maracuyá en Piura. Disponible en: https:// www.infoagro.com/noticias/2009/5/9178_ aumenta_produccion_maracuya_piura.asp.

INTAGRI S.C., (2019). Capacidad de intercambio catiónico. Extraído de https:// www.intagri.com/articulos/suelos/lacapacidad-de-intercam-cat.-del-suelo.

Jalon, M., y Azcon, M. (2008). Fundamentos de Fisisología vegetal. Capítulo 29 Fisiología de las plantas y el estrés. 2da. Edic. Interamericana - Mc Graw - Hill Mdiel pp 577597.

Malavolta, E. (1994). Fertilizantes e seu impacto ambiental: Micronutrientes e metais pesados, mitos, mistificação e fatos. Ed. ProduQuímica. São Paulo, Brasil. 153 p.

Martin, R. (2010). Agrositio Desecho o Aprovechamos el Silicio. Recuperado el 23 de julio de 2013, de Agrositio.

Mondragón. (2004). Comportamiento de tres abonos orgánicos en el rendimiento y calidad del fruto. Alto Piura.

Nilsen, D. (1996). Physiology of plants under stree In: Anbiotic factors. New York: Eds INC.

Osterloh, A., Ebert G., Held W.H., Schulz H., y E. Urban. (1996). Lagerung von Obst und Südfrüchten. Verlag Ulmer, Stuttgart. 253 p.

Pereira, V. (2015). Estudio a la aplicación de tres frecuencias y dos dosis de N-P-K más una fórmula de fertilizante foliar en el cultivo de maracuyá. Guayaquil- Ecuador.

Primavesi, A. (1984). Manejo ecológico del suelo: la agricultura en regiones tropicales. 5 ed. Buenos Aires: Ataneo. 
Quero, E. (2008). Cultivo de maracuyá. Recuperado el 24 de julio de 2013.

Rodríguez, L., López, L., y García, M. (2015). Determinación de la composición química y actividad antioxidante en distintos estados de madurez de frutas de consumo habitual en Colombia, mora (Rubus glaucus b.), maracuyá (Passiflora edulis S.), guayaba (Psidium guajava I.) y papayuela (Carica cundinamarcensis J.). Facultad de Ciencias Naturales, Ingeniería de Alimentos. Universidad Jorge Tadeo Lozano. 